\title{
miR-1207-5p suppresses lung cancer growth and metastasis by targeting CSF1
}

\author{
Wei Dang ${ }^{1,2,3, *}$, Zailong Qin ${ }^{1,2,3, *}$, Songqing Fan ${ }^{4}$, Qiuyuan Wen ${ }^{4}$, Yuanjun Lu' ${ }^{1,2,3}$, Jia \\ Wang ${ }^{1,2,3}$, Xuemei Zhang ${ }^{1,2,3}$, Lingyu Wei ${ }^{1,2,3}$, Wei He ${ }^{2,5}$, Qiurong Ye ${ }^{1,2,3}$, Qun Yan ${ }^{6}$, \\ Guiyuan $\mathbf{L i}^{1,2,3}$, Jian Ma ${ }^{1,2,3}$ \\ ${ }^{1}$ Hunan Cancer Hospital and the Affiliated Cancer Hospital of Xiangya School of Medicine, Central South University, Changsha, \\ Hunan, China \\ ${ }^{2}$ Cancer Research Institute, Central South University, Changsha, Hunan, China \\ ${ }^{3}$ Hunan Key Laboratory of Nonresolving Inflammation and Cancer, Key Laboratory of Carcinogenesis of Ministry of Health, \\ Key Laboratory of Carcinogenesis and Cancer Invasion of Ministry of Education, Changsha, Hunan, China \\ ${ }^{4}$ Department of Pathology, The Second Xiangya Hospital of Central South University, Changsha, Hunan, China \\ ${ }^{5}$ Xi'an Children's Hospital, Xi'an, Shaanxi, China \\ ${ }^{6}$ Department of Clinical Laboratory, Xiangya Hospital of Central South University, Changsha, Hunan, China \\ *These authors contributed equally to this work
}

Correspondence to: Jian Ma, e-mail: majian@csu.edu.cn

Keywords: miR-1207-5p, CSF1, macrophage, tumor microenvironment, lung cancer

Received: November 01, $2015 \quad$ Accepted: March 29, 2016

Published: April 13, 2016

\section{ABSTRACT}

We previously reported that miR-1207-5p can inhibit epithelial-mesenchymal transition (EMT) induced by growth factors such as EGF and TGF- $\beta$, but the exact mechanism is unclear. Here we identified that Colony stimulating factor 1 (CSF1) is a target gene of miR-1207-5p. CSF1 controls the production, differentiation and function of macrophage and promotes the release of proinflammatory chemokines. We showed that miR-1207-5p inhibited lung cancer cell A549 proliferation, migration and invasion in vitro, and suppressed the STAT3 and AKT signalings. miR-1207-5p overexpression can increase HUVEC angiogenesis, and can modulate the M2 phenotype of macrophage. miR-1207-5p also significantly inhibited A549 cells metastasis in a nude mouse xenograft model. miR-1207-5p and CSF1 expression levels and their relationship with lung cancer survival and metastasis status were assayed by means of a lung cancer tissue microarray. Macrophage is an essential part of the tumor microenvironment, thus the miR-1207-5p-CSF1 axis maybe a new regulator of lung cancer development through modulating the tumor microenvironment.

\section{INTRODUCTION}

We previously identified three microRNAs (miR-148a, miR-505 and miR-1207-5p) that provide feedback regulation of epithelial-mesenchymal transition (EMT) induced by growth factors such as EGF and TGF- $\beta$. MicroRNAs (miRNAs) confer robustness to biological processes by targeting key players that are involved in previous development stages. We reported that miR-1207-5p acted as a new negative regulator of EMT, suppressing cancer cell invasion and metastasis in nasopharyngeal carcinoma cells [1]. However, the mechanism of its function in cancer growth and metastasis is still unclear.
There were a few of reports on miR-1207's role in cancer biology. Chen L et al. showed that miR- 1207 suppresses gastric cancer growth and invasion by targeting telomerase reverse transcriptase (hTRET) [2]. MiR-1207-5p was significantly upregulated in gastric cancer patients without lymph node metastasis (LNM) compared with those with LNM [3]. Recently, Wu et al. discovered that miR-1207 directly targetes multiple negative regulators (SFRP1, AXIN2 and ICAT) of Wnt/ $\beta$-catenin signaling and promoted cancer stem cell-like traits in ovarian cancer [4]. So, more target genes are needed to be identified for miR-1207-5p in a variety of cancer types. 
In the current study we identified a novel target gene $C S F 1$ for miR-1207-5p, and investigated its role in tumorigenesis of lung cancer. Colony stimulating factor 1 (CSF1, also known as macrophage (M)-CSF) is a hemopoietic growth factor for the mononuclear phagocyte lineage and the primary regulator of macrophage differentiation, proliferation and survival. CSF1 elicits its effect through binding with CSF1 receptor (CSF1R) which is a high-affinity receptor tyrosine kinase encoded by the $c$-fms proto-oncogene. CSF 1 is secreted by a variety of cell types and acts both locally and humorally in an autocrine and paracrine manner. CSF1 and CSF1R have been reported to be expressed by the tumor epithelium in several human epithelial cancers, including breast, ovarian, lung, endometrial, trophoblastic and prostatic cancer. [5-14] Under this circumstance, we suggested that mR-1207-5p plays an important role in tumor microenvironment through regulating CSF1.

\section{RESULTS}

\section{miR-1207-5p directly targets and inhibits CSF1}

To explore the function of miR-1207-5p in the EMT and metastasis processes, three computational algorithms (TargetScan, PicTar and miRanda) were used to search for potential miR-1207-5p target genes. Among these candidate target genes, CSF1, which was predicted by all three algorithms, attracted our attention. CSF1 is a cytokine that controls the production, differentiation, and function of macrophage. CSF1 Promotes the release of proinflammatory chemokines, and thereby plays an important role in innate immunity and in inflammatory processes. CSF1 expression by macrophages, stromal cells and osteoclasts allows for important paracrine interactions between host microenvironment and cancer cells. Two miR-1207-5p-binding sites were found in the $3^{\prime}$-UTR of CSF1 mRNA, and there were perfect base pairing between the seed sequence of mature miR-1207-5p and the 3'-UTR of CSF 1 mRNA (Figure 1A left). We subcloned the fulllength CSF1 3'-UTR into a luciferase reporter vector. Figure 1A (right) shows that miR-1207 mimics can inhibit the wild-type CSF1 3'-UTR luciferase activity, but the inhibition ability is compromised for two mutant CSF1 3'-UTR vectors. We transfected miR-1207-5p mimics into HEK293, A549, H358, and HK-1 cells, and found that miR-1207-5p mimics reduced CSF1 mRNA levels (Figure 1B).

Next, we used quantitative real-time PCR (qPCR) to measure miR-1207-5p and CSF1 expression levels in different cancer cell lines. Figure $1 \mathrm{C}$ shows that lung adenocarcinoma cell line A549 has relative low miR-1207-5p expression and high CSF1 expression, so, we chose the A549 cell for the in vitro study. Western blotting and ELISA revealed that miR-1207-5p mimics reduced the CSF1 protein levels and its secretion, while
miR-1207-5p inhibitor increased its levels in A549 cells (Figure 1D, 1E). We also transfected expression vector of CSF1-CDS (with or without 3'-UTR) to the A549 cells with miR-1207-5p mimics, respectively, and found that miR-1207-5p only can decrease CSF 1 expression when the expression-vector has CSF1 3'-UTR, which suggested the inhibition of CSF1 expression by miR-1207-5p was dependent on CSF1 3'-UTR (Figure 1F). These results provided evidence that miR-1207-5p directly recognize the 3 '-UTR of CSF 1, and thereby inhibit their translation.

\section{miR-1207-5p inhibits lung cell proliferation and invasion in vitro}

In light of the above findings, we decided to explore the biological significance of miR-1207-5p in lung tumorigenesis. We found that miR-1207-5p mimics markedly attenuated A549 cells proliferation compared to the control group through a colony formation assay (Figure 2A). We also noticed that miR-1207-5p mimics inhibited the in vitro migrative and invasive potentials of A549 cells compared to the control group (Figure 2B, 2C). We also observed similar effect of miR-1207-5p in another lung cancer cell line H358 (Supplementary Figure 1). We previously have revealed that miR-1207-5p can suppress a few of EMT-related molecules [1], and here, we also found that it can downregulate some important EMT molecules, such as Snail, Smad2, Smad3, Smad7, Vimetin, ZEB1 in A549 cells (Supplementary Figure 2).

We next explored whether the miR-1207-5p mimics could affect angiogenesis using an endothelial cell tube formation assay. As shown in Figure 2D, medium from A549 cells overexpressing miR-1207-5p inhibited HUVEC cells tube formation compared to the control group. We also transfected construct encoding CSF1-CDS (with or without 3'-UTR) to the A549 cells with miR-1207-5p mimics, respectively, and found that CSF1 without 3'-UTR, but not the CSF1 with 3'UTR, can significantly increase the angiogenic potential of the cells under the influence of miR-1207-5p overexpression (Figure 2D). We also transfected the HUVEC cells with either miR-1207-5p mimics or control mimics, and found that miR-1207-5p can directly suppress the angiogenic ability of HUVEC cells (Supplementary Figure 3). These observations suggested that miR-1207-5p can suppress the proliferation, migration, and invasion of lung cancer cells in vitro and it also can inhibit endothelial cell tube formation to some extent.

\section{miR-1207-5p inhibits the AKT and STAT3 signaling}

CSF1-CSF1R activation can trigger several important signaling pathways, such as the PI3K-AKT and STAT3 pathways [15-17], most of which regulate cell proliferation, invasion, and inflammation. Therefore, we 
A CSF1 3'UTR-WT GAGGGGAGGCCAGCCCUGCCC miR-1207-5p GGGGAGGGUCGGAGGGACGGU

CSF1 3'UTR-MUt1 GAGGGGAGGCCAGUUAGAUAC
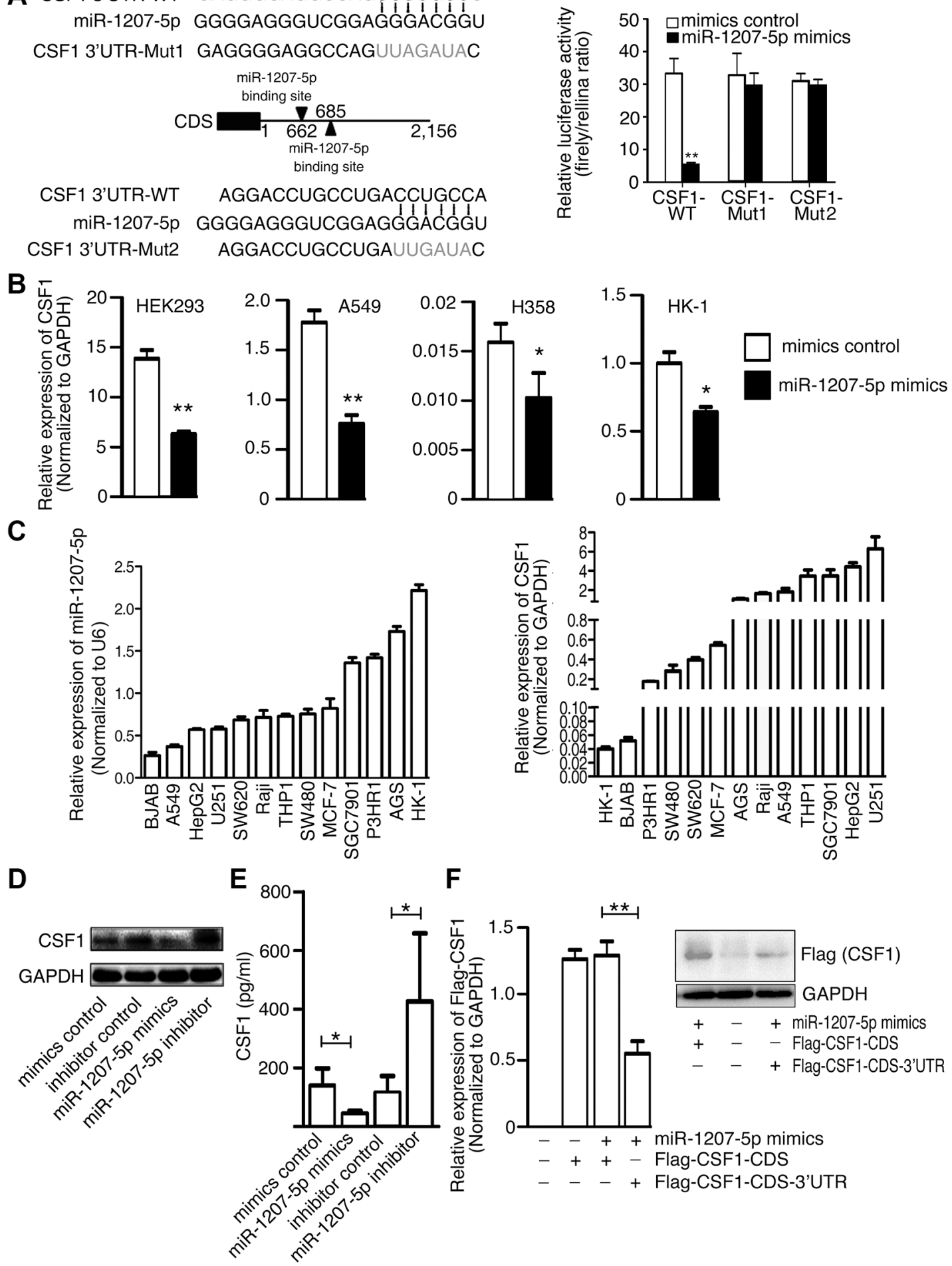

$\mathbf{F}$

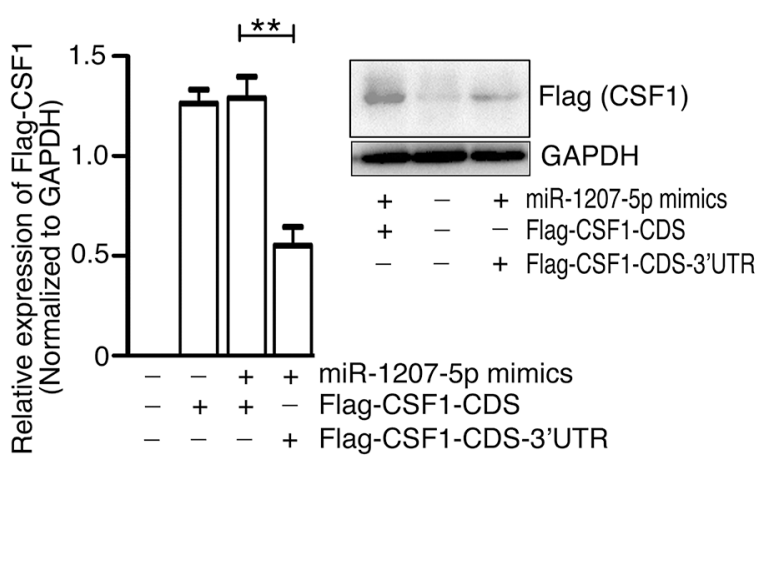

Figure 1: CSF1 is a direct target of miR-1207-5p. (A) Left: Scheme for the potential binding sites of miR-1207-5p in the $3^{\prime}-$ UTR of CSF1, and the sequence of each intact miR-1207-5p binding site (wild-type, WT) and its mutant (Mut) within the luciferase reporter vector. Right: HEK-293 cells were cotransfected with the miR-1207-5p mimics (or control) and a luciferase reporter containing the 3'-UTR (WT or Mut) of CSF1 for 36 hrs. Cell lysates were then harvested for luciferase activities assay. (B) HEK293, A549, H358 and HK-1 cells were transfected with either miR-1207-5p mimics or mimics control for $48 \mathrm{hrs}$, the CSF1 mRNA levels were then analyzed by RT-qPCR. (C) RT-qPCR analysis of the expression levels of CSF1 and miR-1207-5p in multiple cancer cell lines. (D-E) A549 cells were transfected with either miR-1207-5p mimics or inhibitors for $48 \mathrm{hrs}$. CSF1 protein levels in cell lysates were analyzed by Western blotting (D), and in cell supernatant were analyzed by ELISA (E). (F) A549 cells were transfected with indicated vectors and mimics for 48 hrs. Flag-CSF1 mRNA and protein levels were analyzed by RT-qPCR (left) or Western blotting (right). All data are shown as means \pm s.e.m. ${ }^{*} P<0.05$, $* * P<0.01$ compared with control. 
investigated the possibility that miR-1207-5p regulates those pathways by targeting CSF1. miR-1207-5p mimics decreased the phosphorylation levels of AKT and AKT signaling downstream target S6 (Figure 3A). We also observed that miR-1207-5p mimics inhibited phosphorylation levels of STAT3 and its downstream
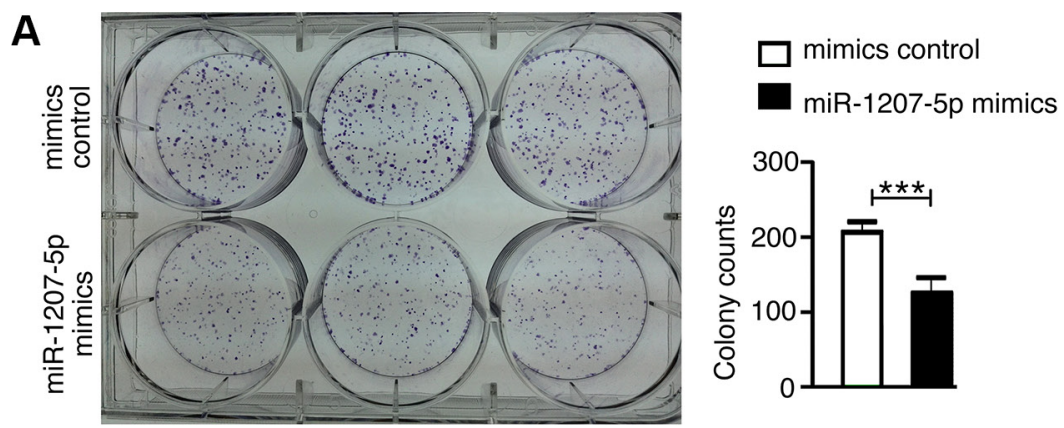

B
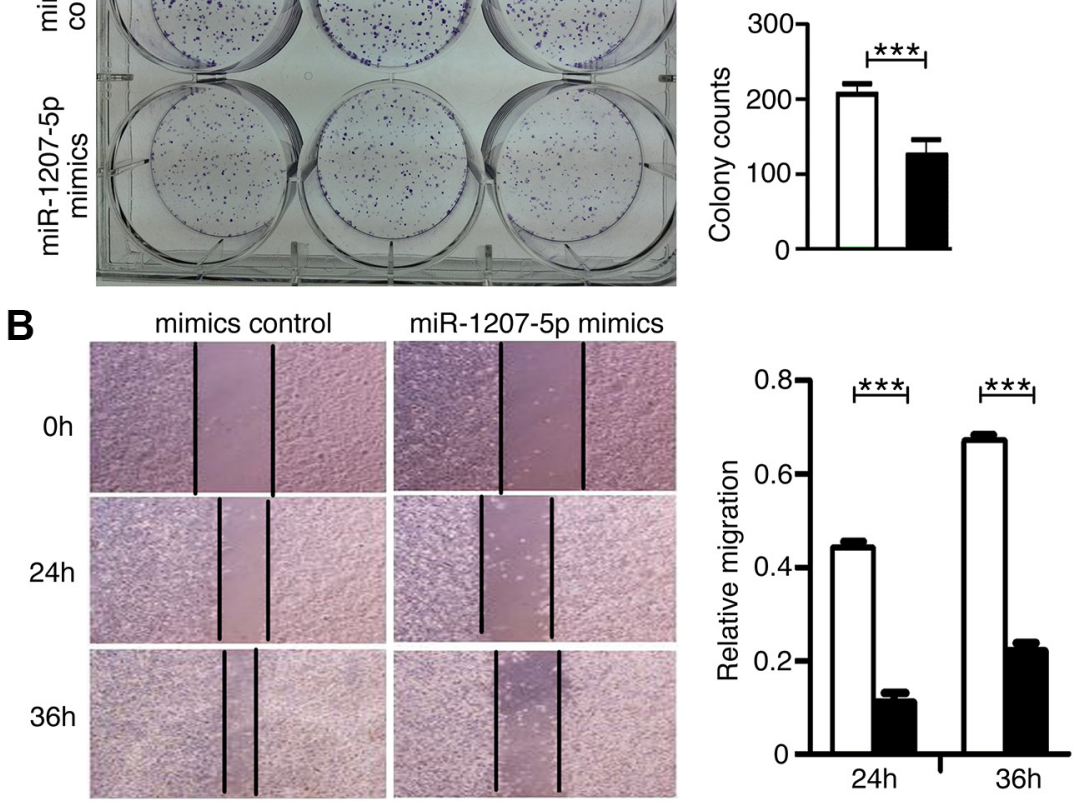

C

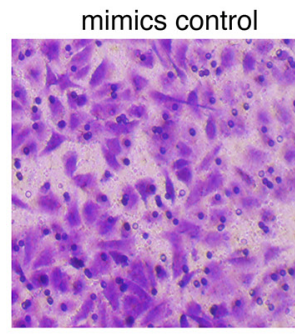

miR-1207-5p mimics
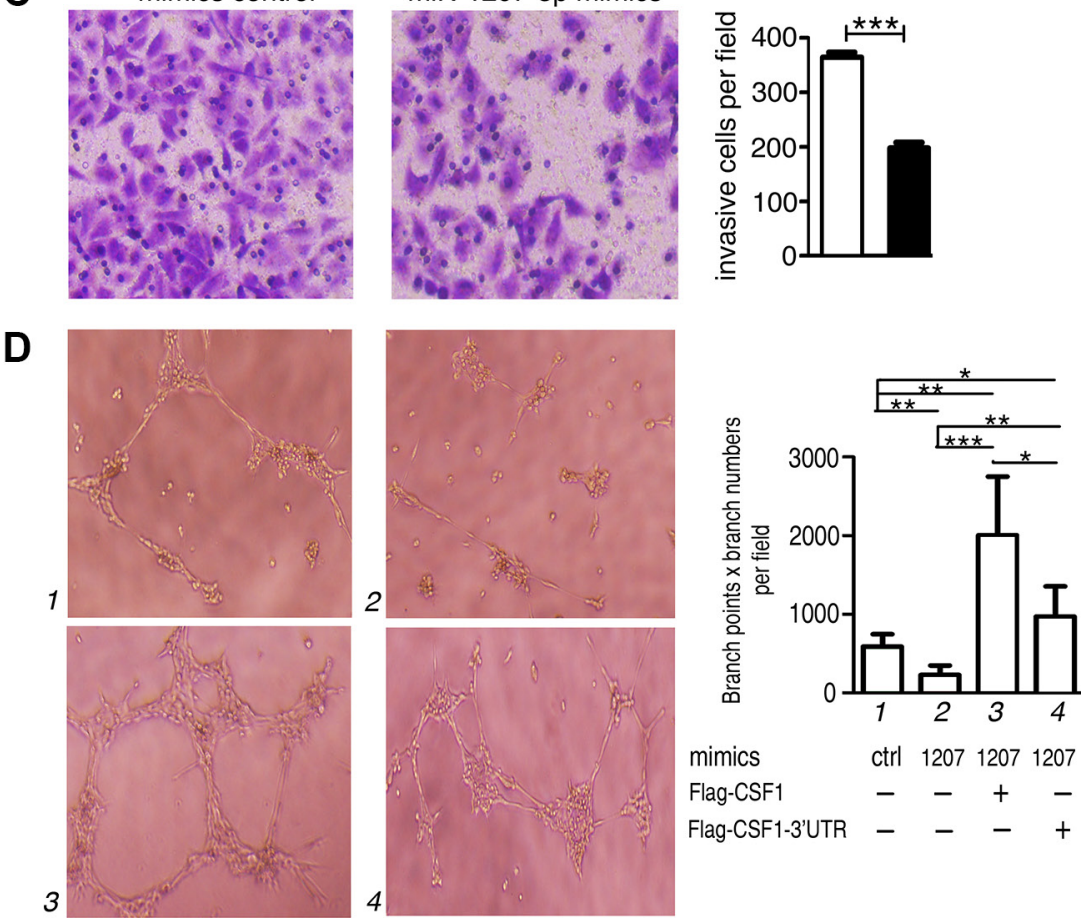

$\begin{array}{lcccc}\text { mimics } & \text { ctrl } & 1207 & 1207 & 1207 \\ \text { Flag-CSF1 } & - & - & + & - \\ \text { Flag-CSF1-3'UTR } & - & - & - & +\end{array}$

Figure 2: miR-1207-5p suppresses the tumorigenicity of A549 cells and inhibit tube formation of endothelial cells in vitro. (A) A549 cells were transfected with either miR-1207-5p mimics or mimics control for $24 \mathrm{hrs,} \mathrm{and} 1000$ cells were seeded into 6-well plates for 7 days to assay the cloning formation ability. (B) A549 cells were transfected with either miR-1207-5p mimics or mimics control for $24 \mathrm{hrs}$, and were seeded into 6-well plates to assay the wound-healing ability. (C) A549 cells were transfected with either miR-1207-5p mimics or mimics control for $24 \mathrm{hrs}$, and were seeded into the insert of transwell to assay the invasion ability. Representative figures of the migrated stained cells are shown. The cells in five randomly selected areas were counted. (D) A549 cells were transfected with indicated mimics or vectors for $48 \mathrm{hrs}$, and then, the cell supernatants were collected and added to the medium of HUVEC to assay their effect on the tube formation ability of HUVEC. All data are shown as the mean \pm s.e.m. ${ }^{*} p<0.05,{ }^{*} p<0.01,{ }^{* * *} p<0.001$ compared with control. 
target genes including CXCL10, CCL5, and IL-10 (Figure 3A, 3B) $[18,19]$. Macrophage plays vital role in the tumor microenvironment [20-22], and CSF1 is essential for proliferation, differentiation, and function of macrophage [6]. We next explored whether the miR-1207-5p mimics could modulate macrophage's function. Macrophage cell d-THP1 was transfected with either miR-1207-5p mimics or control mimics, RT-qPCR of cellular RNA showed that miR-1207-5p can suppress $I L-10$ and $V E G F$ (M2 macrophage characters) expression levels, whereas increase $I L-12$ and $I L-1 B$ (M1 macrophage characters) levels; ELISA of the supernatant also confirmed that miR-1207-5p inhibited IL-10 secretion, whereas promoted IL-12/-23 secretion (Figure 3C).

\section{miR-1207-5p suppresses lung cancer cell metastasis in vivo}

Because overexpression of miR-1207-5p could inhibit tumorigenesis in vitro, we next asked whether it could inhibit the metastatic potential of A549 cells in vivo. A549-luciferase cells that had been treated with
miR-1207-5p agomir or control agomir were injected into the tail vein of nude mice, and the efficiency of transfection was verified (Figure 4A right). Lung metastasis was then examined using the Xenogen IVIS imaging system. High luciferase activity was observed in the lung in the mice receiving the cells transfected with control agomir, whereas significantly reduced luciferase activity was observed in the miR-1207-5p agmoir group (Figure 4A left). The lungs with metastases were resected and processed for H\&E staining, which showed significant decrease of metastases the lungs of in miR-1207-5p agomir group compared to the control group (Figure 4B). Further, immunohistochemical staining revealed that treated with the miR-1207-5p agomir, resulted in decreased expression of CSF1, phos-AKT, and phos-STAT3 in the lung tissues (Figure 4C left). RNA isolated from the lung tumor tissues was used for RT-qPCR assay, and it showed that the miR-1207-5p expression levels were significantly increased in the miR-1207-5p agomir treatment group, whereas CSF1 expression is decreased compared with control group (Figure $4 \mathrm{C}$ right). These results indicated that miR-1207-5p suppressed the metastasis of A549 cells to lung in vivo.
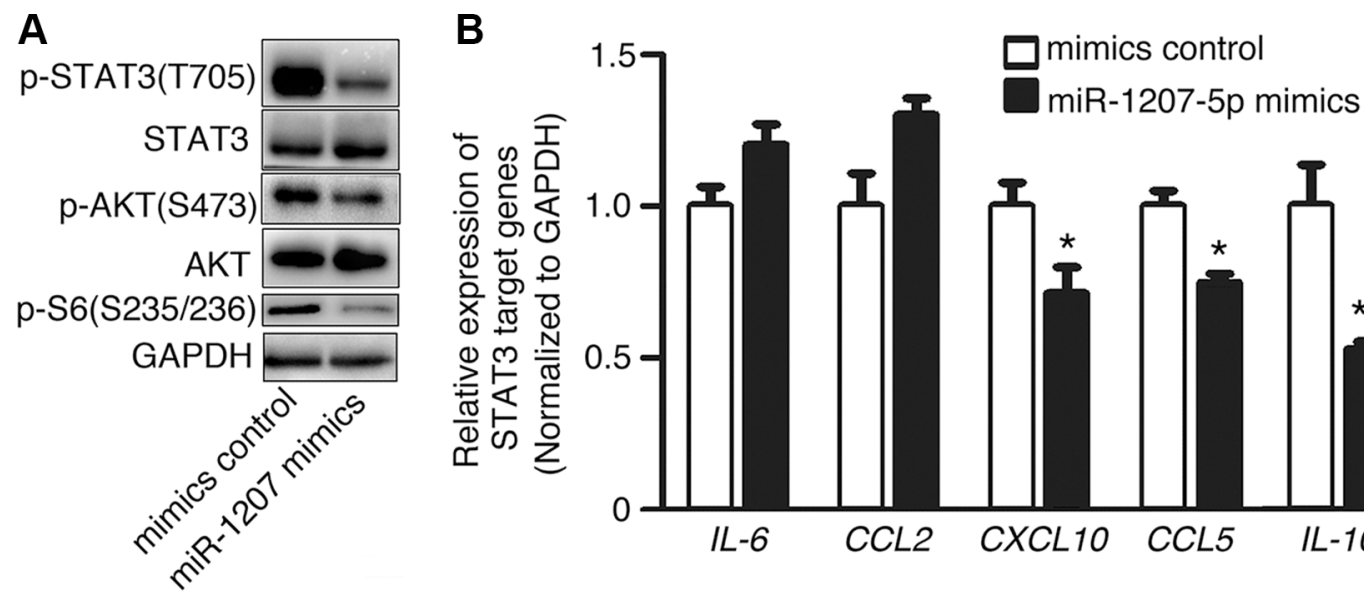

C
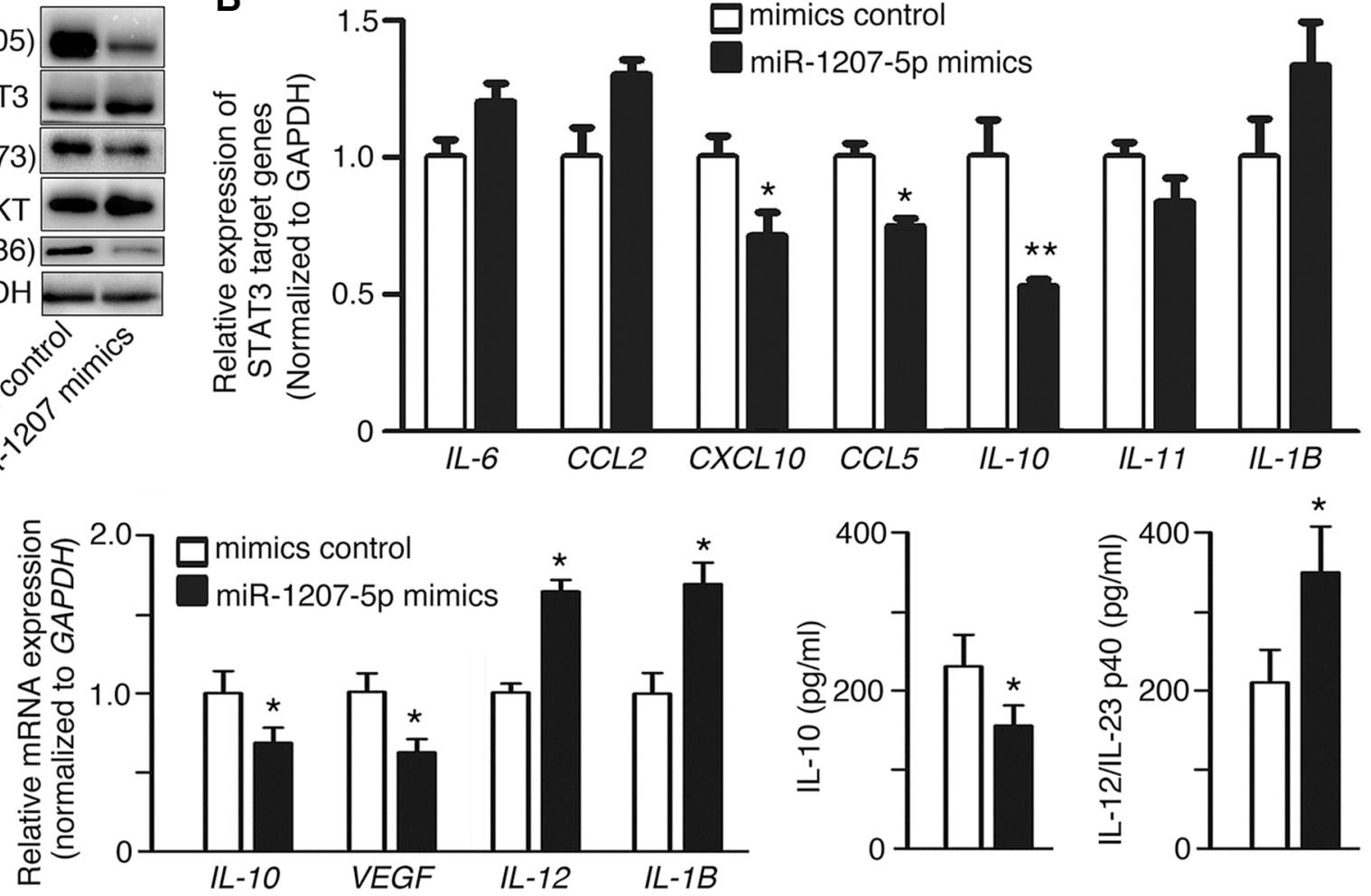

Figure 3: miR-1207-5p inhibits the AKT and STAT3 signalings. (A-B) A549 cells were transfected with either miR-1207-5p mimics or control mimics for $48 \mathrm{hrs}$, and the cellular proteins and RNAs were isolated. The cellular protein levels of p-STAT3, p-AKT, p-S6 were assayed by Western blotting (A). The STAT3 signaling downstream target genes (IL-6, CCL2, CXCL-10, CCL5, IL-10, IL$11, I L-1 B)$ mRNA expression levels were assayed by RT-qPCR (B). (C) To assay the effect of miR-1207-5p on macrophage characters, d-THP1 cells were transfected with either miR-1207-5p mimics or control mimics for $48 \mathrm{hrs,} \mathrm{then,} \mathrm{total} \mathrm{RNA} \mathrm{was} \mathrm{isolated} \mathrm{and} \mathrm{RT-qPCR}$ was performed to assay the M1/M2 macrophage phenotype-related genes expression levels (left); and IL-10 and IL-12/23 protein levels from cell supernatant were assayed by ELISA (right). All data are shown as the mean \pm s.e.m. ${ }^{*} p<0.05,{ }^{*} p<0.01$ compared with control. 
A

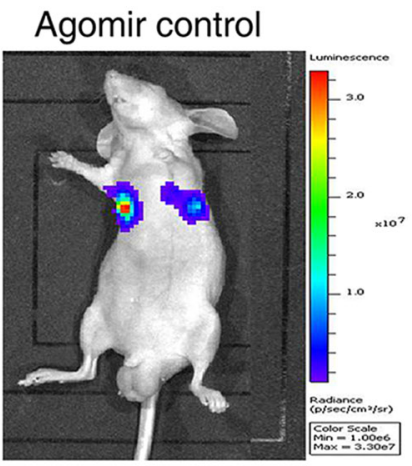

B

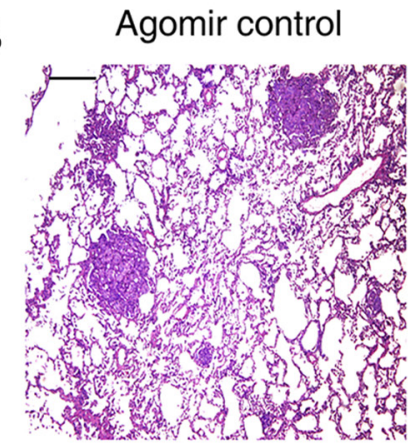

C

Agomir control
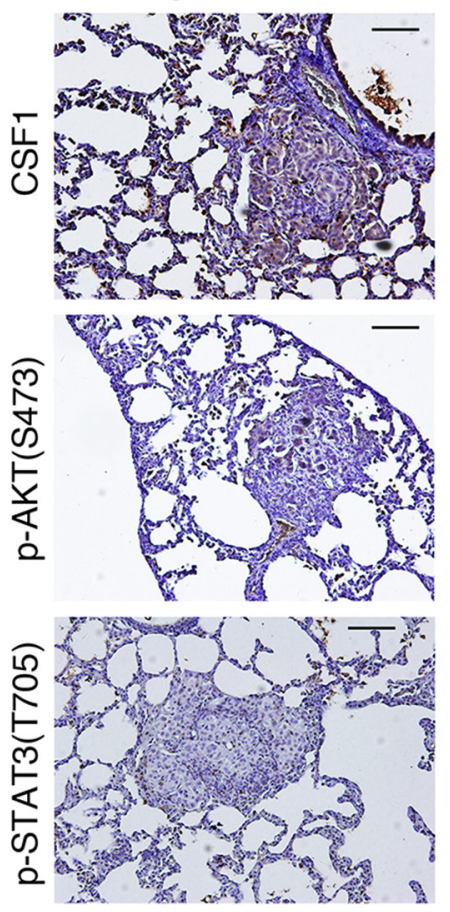
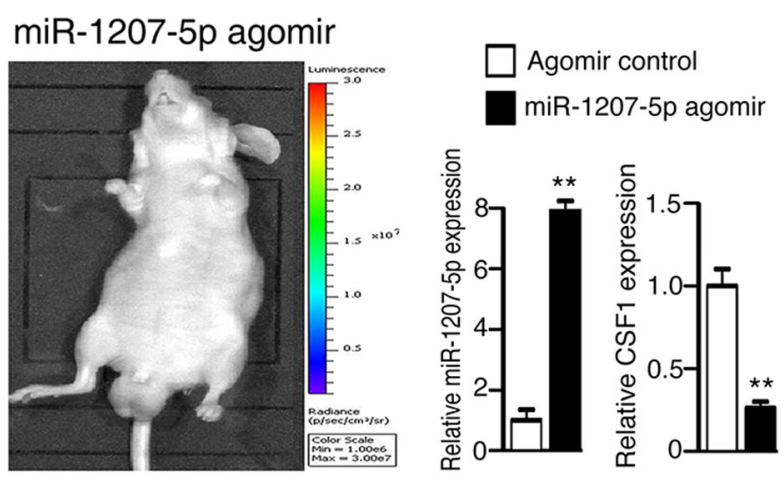

miR-1207-5p agomir
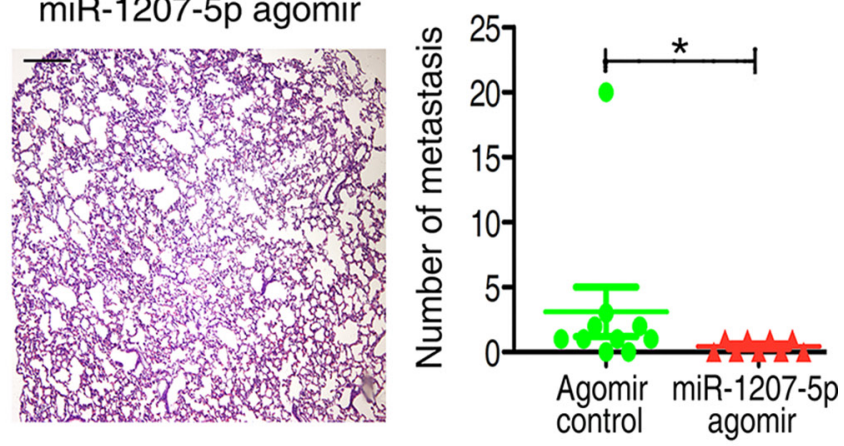

miR-1207-5p agomir
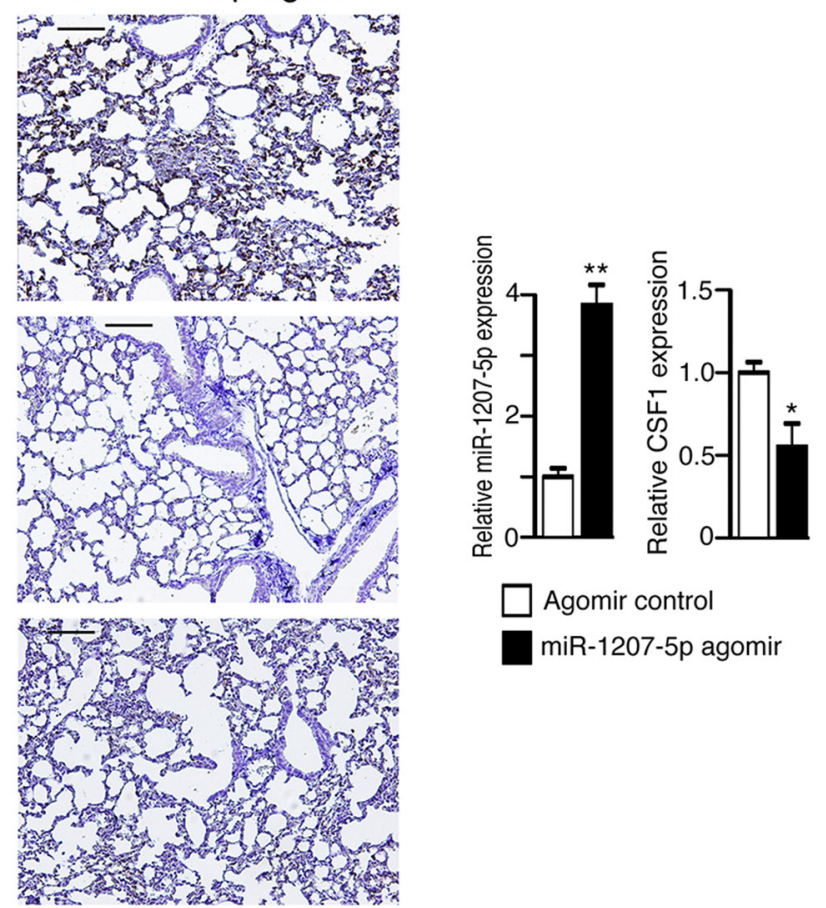

Figure 4: miR-1207-5p suppresses the metastasis of A549 cells in a nude mouse xenograft model. The tail veins of nude mice were intravenously injected with A549-luciferase cells (which had been treated with either miR-1207-5p agomir or a control agomir for $24 \mathrm{hrs}$ ). miR-1207-5p agomir group has nine mice and control agomir group has ten mice. After 7 days, each group of nude mice were treated with miR-1207-5p agomir or control agomir through vein injection, respectively. Fifty days later, D-Luciferin was administered to each mouse, and the mice were then imaged by using a Xenogen IVIS Lumina II imaging system. After then, necropsies and pathology examination were performed. (A) Representative images of IVIS imaging, which revealed the metastatic nodules in the lung (left). Effects of miR-1207-5p agomir on miR-1207-5p and CSF1 mRNA expression in A549 cells assayed by RT-qPCR (right). (B) Representative images of H\&E-stained mice lung sections, which revealed the metastatic nodules (left), and numbers of metastases in lungs of individual mice were analyzed (right). (C) The expression of CSF1, p-AKT and p-STAT3 were assayed by immunohistochemistry (left). The mRNA levels of miR-1207-5p and CSF1 of mice lung tumor tissues were analyzed by RT-qPCR (right). Scale bar: $50 \mu \mathrm{m}$. All data are shown as the mean \pm s.e.m. ${ }^{*} p<0.05, * * p<0.01$ compared with control. 
miR-1207-5p and CSF1 expression levels are associated with clinicopathological parameters of NSCLC patients

Lung cancer is a major cause of cancer-related mortality worldwide, and $80 \%-85 \%$ of lung cancer cases diagnosed with non-small cell lung cancer (NSCLC), among which adenocarcinoma (ADC) and squamous cell carcinoma (SCC) are the two major histological subtypes [23]. In this study, we examined miR-1207-5p mRNA expression levels and CSF1 protein expression levels by analyzing tissue microarray containing lung ADC $(n=151)$ and SCC $(n=138)$ specimens as well as non-cancerous lung tissues $(n=53)$. Strong positive expression of miR-1207-5p was identified on cytoplasm of noncancerous lung tissues, and much lower expression were detected in ADC and SCC. In contrast, strong positive expression of CSF1 was identified in the cytoplasm of cancer tissues, but only weak staining was observed in the non-cancerous lung tissues (Figure 5A, 5B). In this tissue microarray, there are 118 patients had primary cancer tissues as well as metastases tissues. We found that miR1207-5p expression levels were decreased in metastases tissues relative to their primary tissues. In contrast, the CSF1 expression levels were higher in metastases compared with their primary tissues (Figure 5C).

In univariate survival analysis of NSCLC patients, Kaplan-Meier survival curve analysis with log-rank significance test was performed. The overall survival rates for NSCLC patients with high expression of miR-1207-5p were significantly higher than these with low miR-1207-5p expression ( $P=0.01$, Figure 5D left). However, no significant association between expression of CSF1 and overall survival rates was noticed in this cohort patients $(P>0.05$, Figure 5D middle). We next used MIRUMIR (a database for survival analyses in cancer [24]) and analyzed the clinic impact of CSF1 expression patterns on lung cancer patients survival. In a NSCLC cancer specimen expression profile dataset (GSE31210, [25]), cancer with low expression of CSF1 had better survival chance compared with cancer with high expression of $C_{S F 1}(P=0.04$, Figure 5D right). The human NSCLC data supported the concept that miR-1207-5p has anti-metastasis or tumor suppressor functions, whereas CSF1 has pro-metastasis or oncogene function.

\section{DISCUSSION}

In order to discovery new miRNAs involved in the EMT process, we previously used EGF or TGF- $\beta$-induced EMT cellular model, and identified that miR-1207-5p is induced by both EGF and TGF- $\beta$ and functions as negative feedback regulator of EMT [1]. There are limited information on miR-1207-5p's role in tumorigenesis and metastasis. In this study, we identified a novel target gene
CSF1 for miR-1207-5p. miR-1207-5p can inhibit CSF1 $\mathrm{mRNA}$ /protein expression and secretion in lung cancer cell A549 by targeting the 3 '-UTR of the CSF 1 mRNA.

As a growth factor, CSF1 is secreted by macrophages, epithelial and fibroblasts cells and cancer cells [26]. CSF1 promotes monocyte differentiating into macrophage, and support macrophage survival and proliferation. CSF 1 and its receptor CSF1R play vital roles in normal development, and function as mediators of intercellular communication by diffusible molecules [27]. CSF1 can stimulate cancer cells directly, and it also can promote tumorigenesis indirectly through mobilizing and adjusting the host immune system responding to cancer cells. Tumor associated macrophages (TAMs) stimulated by CSF1 have a wide range of activities like promoting tumor growth, angiogenesis, extracellular matrix breakdown. High microvessel density has a stronger association with expression of CSF1 [28, 29], suggesting that CSF1 may mediate a pro-angiogenesis phenotype. CSF1 promotes the proliferation of TAMs and supports it to produce a few of pro-angiogenic factors including VEGF, bFGF, TNF- $\alpha$, and others [30-32]. The TAM and those factors are important parts of the tumor microenvironment, regulating cancer development, which is called the M2 phenotype of macrophage (usually expressing high levels of IL-10, VEGF and Arg-1; whereas expressing low levels of IL-12, IL-23 and iNOS) [20, 21]. CSF1 related TAM secrets multiple cytokines and promote nonresolving inflammation, and it also degrades the ECM by secreting matrix-bound growth factors [33-35]. CSF1 of the cancer cells can increase the migration ability of macrophages through autocrine loop, thus promotes the tumor invasion and metastasis, and further stimulates the secretion of CSF1 [36].

We reported that miR-1207-5p can suppress lung cancer cell A540 proliferation, migration and invasion. We also discovered that miR-1207-5p can inhibit HUVEC tube formation, which is dependent on the CSF1 3'-UTR existing. Since STAT3 and AKT are two important signaling pathways downstream of CSF1/CSF1R, we assayed the effect of miR-1207-5p on these two pathways. It showed that miR-1207-5p can downregulate STAT3 and AKT signalings, as well as their downstream targets, including some important inflammatory mediators such as IL-10,CCL5 and CXCL10. We also showed that miR-1207-5p can regulate a few of important EMT-related molecules such as Snail, Smad 2, Smad3, Smad7, Vimentin, and ZEB1, which indicated the inhibition role of miR-1207-5p in tumor invasion and metastasis.

We evaluated the effect of miR-1207-5p on macrophage function in d-THP1 cells. miR-1207-5p mimics significantly reduced the $\mathrm{M} 2$ macrophage characters (such as IL-10 and VEGF), whereas increased the M1 macrophage characters (such as IL-12 and IL-23). These results provide evidence that miR-1207-5p could modulate macrophage function of the tumor microenvironment. 
We also used nude mouse xenograft model to confirm that miR-1207-5p could suppress lung cancer cells metastasis in vivo. Immunohistochemistry staining showed that miR-1207-5p suppressed CSF1, phos-AKT, and phos-STAT3 expression in the mouse model.

At last, we found that miR-1207-5p expression levels were significantly downregulated in NSCLC specimens, whereas CSF1 expression levels were upregulated in NSCLC compared to the non-cancerous lung tissues. Also, miR-1207-5p expression levels were lower in metastases compared to their primary tissues, whereas CSF1 had the opposite effect. More importantly, high expression of miR-1207-5p or low expression of CSF1 provided better survival chance for NSCLC patients compared with cancer with low expression of miR-1207-5p or high expression of CSF1 (Figure 5D left and right). These clinic data implies the miR-1207-5p-CSF1 axis may play roles in the NSCLC development in vivo.

Tumor microenvironment provides multiple supports for tumor development and metastasis [34]. Our present study provides new information that miR-1207-5p can target CSF1, an essential growth factor for macrophage, and thus modulate the tumor microenvironment. miR-1207-5p is an anti-metastasis microRNA, and understanding the miR-1207-5p-CSF1 axis will provide additional opportunities for the design of treatments for NSCLC. The exact role of miR-1207-5p in carcinogenesis is worthy of further study.
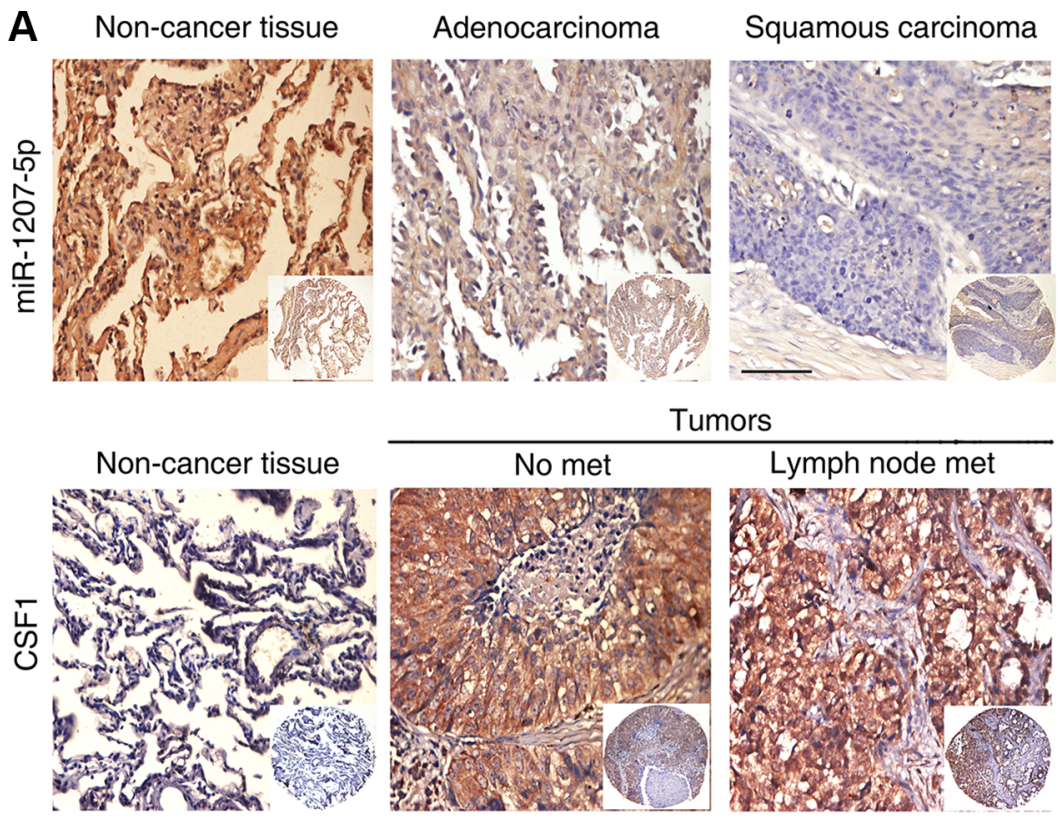

Tumors
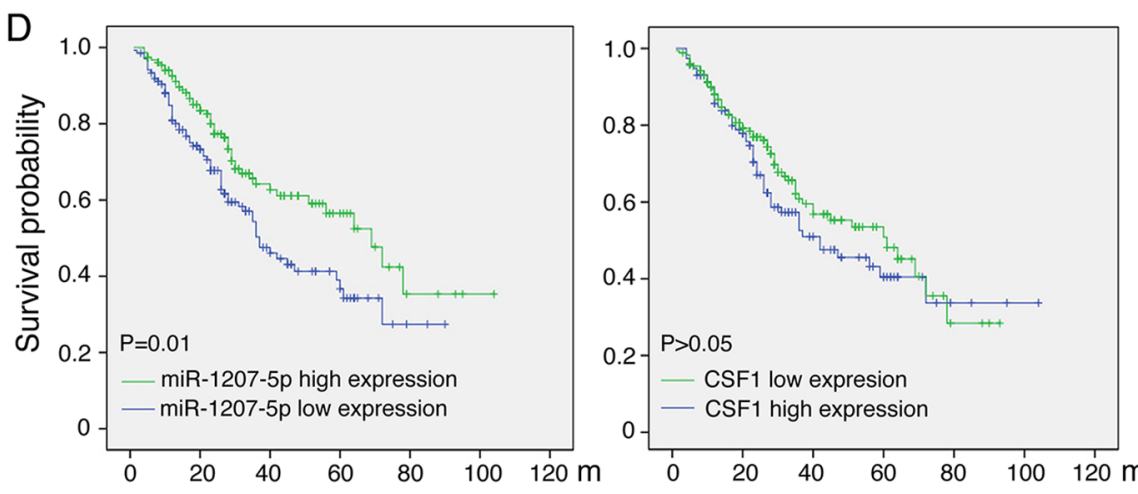

Figure 5: Expression of miR-1207-5p and CSF1 in NSCLC and control non-cancerous lung tissues by means of TMA. (A) In situ hybridization staining of miR-1207-5p mRNA in adenocarcinoma and squamous carcinoma tissues as well as non-cancerous lung tissues (upper panel). Immunohistochemical staining of CSF1 proteins in lung cancer tissues (with or with out lymph node metastasis) and control non-cancerous lung tissues (bottom panel). Scale bar: $50 \mu \mathrm{m}$. (B) Expression scores of miR-1207-5p and CSF1 in lung cancer tissues $(n=289)$ and non-cancerous lung tissues $(n=53)$. (C) Expression scores of miR-1207-5p and CSF1 in lung cancer tissues, which had both primary tumor tissues and metastasis tumor tissues $\left(n=118\right.$ pairs). All data are shown as the mean \pm s.e.m. ${ }^{*} p<0.05, * * p<0.01$, $* * * P<0.001$ compared with control. (D) Kaplan-Meier analysis was used to plot the overall survival curves of 289 cases of NSCLC patients with different expression of miR-1207-5p and CSF1, which statistical significance was assessed by log-rank test (left and middle panel). MIRUMIR database [24] was used to analyze the clinic impact of CSF1 expression patterns on lung cancer patients' survival in a NSCLC cancer specimen expression profile dataset (GSE31210, $n=222)$ (right panel). 


\section{MATERIALS AND METHODS}

\section{Vectors construction}

The 3'-UTR of CSF1 was amplified and subcloned into the pMIR-Report luciferase vector (Ambion, Austin, TX, USA). Mutants of the seed region of the putative miRNA-binding sites in 3'-UTR were generated by point mutation PCR. The CDS and CDS plus 3'UTR of CSF1 were obtained by PCR from CSF1 plasmid (Origene, Rockville, MD, USA) and subcloned into the pCMV-NFlag vector (Beyotime Biotechnology, Jiangsu, China).

\section{Cell culture}

HEK293, A549, H358, HK-1, BJAB, Raji, P3HR1, THP1, HepG2, U251, SW620, SW480, MCF-7, SGC7901 and AGS cells were grown in RPMI 1640 medium supplemented with $10 \%$ feal calf serum (FBS) at $37^{\circ} \mathrm{C}$ with $5 \% \mathrm{CO}_{2}$. A549 and $\mathrm{H} 358$ are human NSCLC cell lines, and HK-1 is a human nasopharyngeal carcinoma cell line. BJAB, Raji and P3HR1 are B cell lymphoma cell lines, and HepG2 is a hepatocellular carcinoma cell line. U251 is a brain glioblastoma cell line, and MCF-7 is a breast cancer cell line. SGC7901 and AGS are gastric cancer cell lines, and SW480 and SW620 are colon cancer cell lines.

To obtain macrophage-like differentiated cells (d-THP1), human monocytic cell line THP-1 cells were treated with $25 \mathrm{ng} / \mathrm{ml}$ phorbol myristate acetate in the medium for $48 \mathrm{~h}$. After the incubation, the phorbol myristate acetate-containing medium was aspirated and adherent differentiated cells were resuspended in fresh medium and incubated for an additional $24 \mathrm{~h}$.

To assay the effect of miR-1207-5p on macrophage characters, d-THP1 cells were transfected with either miR-1207-5p mimics or control mimics for $48 \mathrm{~h}$. Total RNA was purified using Trizol (Life Technologies, Gaithersburg, MD, USA) and RT-qPCR was performed to assay the M1/M2 macrophage phenotype-related genes expression levels.

\section{Quantitative real-time RT-PCR}

Quantitative real-time RT-PCR was carried out as described previously [1]. The primer sequences are listed in the Supplementary Table 1.

\section{Luciferase activity assay}

Luciferase activity assay was carried out as described previously [1].

\section{Wound-healing assay}

A549 or H358 cells were transfected with a synthetic microRNA mimics (GenePharma, Shanghai, China) and then grown in a 6-well plate. After the cell monolayer had reached $90 \%$ confluence, a wound was made with a $10-\mu l$ pipette tip. Cells were then cultured in medium with $1 \%$ FBS, and migration at the corresponding wound site was documented using a microscope at the indicated time points.

\section{Matrigel invasion assay}

Before cell seeding, 24-well Transwell plates (8- $\mu \mathrm{m}$ pores; Corning, New York, NY, USA) were coated with Matrigel matrix (BD Biosciences, San Diego, CA, USA). $5 \times 10^{4}$ transfected A549 or H358 cells suspended in $200 \mu 1$ volume of RMPI 1640 (with 1\% FBS) were added to the top of each insert well. Medium with $15 \%$ FBS was placed in the bottom wells. The cells were then allowed to migrate for $48 \mathrm{~h}$ at $37^{\circ} \mathrm{C}$. The invasived cells were fixed with $10 \%$ methanol for 15 minutes. Then the invasive cells on the lower surface of the membrane were stained with $2 \%$ crystal violet for $5 \mathrm{~min}$, and the stained cells were counted under a microscope. To minimize bias, at least five fields with $100 \times$ magnification were counted, and the various counts were averaged.

\section{Colony formation assay}

A549 or H358 cells were transfected with miRNA mimics and then grown in the 6-well plates for $24 \mathrm{~h}$. For each group, 1000 cells were seeded in triplicate into 6-well plates for 7 days during which period the medium was not changed. Cells were then fixed with $4 \%$ formaldehyde and stained with crystal violet for 5 minutes and washed with PBS for three times.

\section{Tube formation assay}

The tube formation assay was carried out as described [37]. In brief, 96-well plates were coated with $50 \mu$ l Matrigel. $2 \times 10^{4}$ HUVEC cells were added to each well and incubated at $37^{\circ} \mathrm{C}$ for $6-8 \mathrm{~h}$. Images were acquired under an inverted microscope. Antiangiogenic activity was quantified by measuring the length of tube walls formed between discrete endothelial cells in each well relative to the control.

\section{Western blot analysis}

Western blotting was carried out as described previously [38]. Antibodies against phos-STAT3(T705) and phos-S6(S235/236) were obtained from Cell Signaling Technology (Danvers, MA, USA). Anti-CSF1, AKT, and phos-AKT (S473) antibodies were from Abcam (Cambridge, MA, USA). Anti-STAT3 antibody was from Abzoom (Dallas, TX, USA). Anti-Flag antibody was from Sigma-Aldrich (St. Louis, MO, USA). Anti-GAPDH antibody was from Millipore (Billerica, MA, USA). 
Table 1: 289 cases of non small lung cancer (NSCLC) patient's characteristics

\begin{tabular}{|c|c|}
\hline Patients characteristics & No. of cases (\%) \\
\hline \multicolumn{2}{|l|}{ Age (years) } \\
\hline$\leq 50$ & $75(26 \%)$ \\
\hline$>50$ & $214(74 \%)$ \\
\hline \multicolumn{2}{|l|}{ Gender } \\
\hline Male & $217(75 \%)$ \\
\hline Female & $72(25 \%)$ \\
\hline \multicolumn{2}{|l|}{ Clinical stages } \\
\hline $\mathrm{I}$ & $75(26 \%)$ \\
\hline II & $69(24 \%)$ \\
\hline III & $134(46 \%)$ \\
\hline IV & $11(4 \%)$ \\
\hline \multicolumn{2}{|l|}{ LNM status } \\
\hline LNM & $184(64 \%)$ \\
\hline No LNM & $105(36 \%)$ \\
\hline \multicolumn{2}{|l|}{ Histological type } \\
\hline $\mathrm{SCC}$ & $138(48 \%)$ \\
\hline $\mathrm{ADC}$ & $151(52 \%)$ \\
\hline \multicolumn{2}{|l|}{ Histological grades } \\
\hline Well differentiation & $5(2 \%)$ \\
\hline Moderate differentiation & $125(43 \%)$ \\
\hline Poor differentiation & $159(55 \%)$ \\
\hline
\end{tabular}

Abbreviations:

LNM, lymph node metastasis; SCC, squamous cell carcinoma; ADC, adenocarcinoma.

\section{ELISA}

Secretion of CSF1 was detected according to the ELISA Kit for Colony Stimulating Factor 1 assay manual (Cloud-Clone Corp, Houston, USA) after miR-1207-5p mimics or inhibitors were transfected into A549 cells for 48 h. Secretion of IL-10, IL-12/IL-23 was detected by ELISA Kits (purchased from R\&D, Minneapolis, MN, USA) after miR-1207-5p mimics or control mimics were transfected into d-THP1 cells for $48 \mathrm{~h}$.

\section{Animal experiment}

A549-luciferase cells were harvested after treatment of miR-1207-5p agomir or control agomir (Ribobio, Guangzhou, China) for 24h, and single cell suspensions of $8 \times 105$ cells were used for vein injections of four-week-old male athymic BALB/c nude mice. miR-1207-5p agomir group has nine mice and control agomir group has ten mice. Seven days later, each group of nude mice were treated with miR-1207-5p agomir or control agomir $(150 \mu \mathrm{l}, 200 \mathrm{nM})$ through vein injection, respectively. Fifty days later, D-Luciferin was administered to each mouse by intraperitoneal injection at a dose of $150 \mathrm{mg} / \mathrm{kg}$, and the mice were anesthetized for $5 \mathrm{~min}$ in a chamber with $3 \%$ isofluorane. The mice were then imaged by using a Xenogen IVIS Lumina II imaging system (Caliper Life Sciences, Hopkinton, MA, USA). After then, necropsies were performed. After physical separation of the lobes of each lung, the metastases numbers on the surface of each of the five lobes were counted under a dissecting microscope. Tumor cells in lung metastases in individual mice were analyzed by immunohistochemistry. All animal procedures were performed in accordance with institutional guidelines. Agomir is chemically-modified double-strand miRNA mimics which can mimic mature endogenous miRNAs after transfection into cells.

\section{Tissue microarray (TMA) and clinical data}

TMAs were constructed as described previously [39]. Samples were obtained with informed consent and all protocols were approved by The Second Xiangya Hospital of Central South University Ethics Review Board. Written informed consent was obtained from all patients. There are 289 cases of NSCLC patient lung cancer tissues (See Table 1), and 53 non-cancerous lung tissues in the 
TMA. Among the 289 cancer samples, 118 samples had both primary tumor tissues and metastasis tumor tissues.

\section{Immunohistochemistry and scores}

Immunohistochemistry staining and scores were carried out as described previously [39].

\section{In situ hybridization}

In situ hybridization was carried out as describied prebiously [40]. 5'DIG-labeled has-miR-1207-5p miRCURY LNA detection probe was obtained from Exiqon (Vedbaek, Denmark).

\section{Statistical analysis}

Statistical analysis was performed using SPSS17.0 and Graph-Pad Prism 5. Chi-square test or Kruskal-Wallis $H$ test was used for categorical variables. Kaplan-Meier analysis was performed for overall survival curves and statistical significance was assessed using the log-rank test. Independent $t$-test or ANOVA was performed for qRT-PCR and phenotype analyses. Significance parameters were set at $p<0.05$.

\section{FUNDINGS}

This work was supported by National Natural Science Foundation of China (81472694, 81171988), China 111 Project (111-2-12), Ministry of Education (NCET-11-0520), Hunan Provincial Natural Science Foundation of China (14JJ2022), Fundamental Research Funds for the Central Universities of Central South University (2015zzts100).

\section{CONFLICTS OF INTEREST}

The authors declare no conflicts of interest.

\section{REFERENCES}

1. Qin Z, He W, Tang J, Ye Q, Dang W, Lu Y, Wang J, Li G, Yan Q, Ma J. MicroRNAs Provide Feedback Regulation of Epithelial-Mesenchymal Transition Induced by Growth Factors. J Cell Physiol. 2016; 231:120-129.

2. Chen L, Lu MH, Zhang D, Hao NB, Fan YH, Wu YY, Wang SM, Xie R, Fang DC, Zhang H, Hu CJ, Yang SM. miR-1207-5p and miR-1266 suppress gastric cancer growth and invasion by targeting telomerase reverse transcriptase. Cell Death Dis. 2014; 5:e1034.

3. Huang KH, Lan YT, Fang WL, Chen JH, Lo SS, Li AF, Chiou SH, Wu CW, Shyr YM. The correlation between miRNA and lymph node metastasis in gastric cancer. Biomed Res Int. 2015; 2015:543163.

4. Wu G, Liu A, Zhu J, Lei F, Wu S, Zhang X, Ye L, Cao L, He S. MiR-1207 overexpression promotes cancer stem cell- like traits in ovarian cancer by activating the Wnt/beta-catenin signaling pathway. Oncotarget. 2015; 6:28882-28894. doi: 10.18632/oncotarget.4921.

5. Chockalingam S, Ghosh SS. Macrophage colonystimulating factor and cancer: a review. Tumour Biol. 2014; 35:10635-10644.

6. Jones CV, Ricardo SD. Macrophages and CSF-1: implications for development and beyond. Organogenesis. 2013; 9:249-260.

7. Metcalf $\mathrm{D}$. The colony-stimulating factors and cancer. Nat Rev Cancer. 2010; 10:425-434.

8. Chambers SK, Ivins CM, Kacinski BM, Hochberg RB. An unexpected effect of glucocorticoids on stimulation of $\mathrm{c}$-fms proto-oncogene expression in choriocarcinoma cells that express little glucocorticoid receptor. Am J Obstet Gynecol. 2004; 190:974-985.

9. Ide H, Seligson DB, Memarzadeh S, Xin L, Horvath S, Dubey P, Flick MB, Kacinski BM, Palotie A, Witte ON. Expression of colony-stimulating factor 1 receptor during prostate development and prostate cancer progression. Proc Natl Acad Sci U S A. 2002; 99:14404-14409.

10. Filderman AE, Bruckner A, Kacinski BM, Deng N, Remold HG. Macrophage colony-stimulating factor (CSF-1) enhances invasiveness in CSF-1 receptor-positive carcinoma cell lines. Cancer Res. 1992; 52:3661-3666.

11. Kacinski BM, Scata KA, Carter D, Yee LD, Sapi E, King BL, Chambers SK, Jones MA, Pirro MH, Stanley ER, et al. FMS (CSF-1 receptor) and CSF-1 transcripts and protein are expressed by human breast carcinomas in vivo and in vitro. Oncogene. 1991; 6:941-952.

12. Kacinski BM, Chambers SK, Stanley ER, Carter D, Tseng P, Scata KA, Chang DH, Pirro MH, Nguyen JT, Ariza A, et al. The cytokine CSF-1 (M-CSF) expressed by endometrial carcinomas in vivo and in vitro, may also be a circulating tumor marker of neoplastic disease activity in endometrial carcinoma patients. Int J Radiat Oncol Biol Phys. 1990; 19:619-626.

13. Kacinski BM, Carter D, Mittal K, Yee LD, Scata KA, Donofrio L, Chambers SK, Wang KI, Yang-Feng T, Rohrschneider LR, et al. Ovarian adenocarcinomas express fms-complementary transcripts and fms antigen, often with coexpression of CSF-1. Am J Pathol. 1990; 137:135-147.

14. Rettenmier CW, Sacca R, Furman WL, Roussel MF, Holt JT, Nienhuis AW, Stanley ER, Sherr CJ. Expression of the human c-fms proto-oncogene product (colonystimulating factor-1 receptor) on peripheral blood mononuclear cells and choriocarcinoma cell lines. J Clin Invest. 1986; 77:1740-1746.

15. Li L, McBride DW, Doycheva D, Dixon BJ, Krafft PR, Zhang JH, Tang J. G-CSF attenuates neuroinflammation and stabilizes the blood-brain barrier via the PI3K/Akt/ GSK-3beta signaling pathway following neonatal hypoxiaischemia in rats. Exp Neurol. 2015.

16. Cui YH, Suh Y, Lee HJ, Yoo KC, Uddin N, Jeong YJ, Lee JS, Hwang SG, Nam SY, Kim MJ, Lee SJ. Radiation promotes invasiveness of non-small-cell lung cancer cells 
through granulocyte-colony-stimulating factor. Oncogene. 2015; 34:5372-5382.

17. Agarwal S, Lakoma A, Chen Z, Hicks J, Metelitsa LS, Kim ES, Shohet JM. G-CSF Promotes Neuroblastoma Tumorigenicity and Metastasis via STAT3-Dependent Cancer Stem Cell Activation. Cancer Res. 2015; 75:2566-2579.

18. Nguyen PM, Putoczki TL, Ernst M. STAT3-Activating Cytokines: A Therapeutic Opportunity for Inflammatory Bowel Disease? Journal of interferon \& cytokine research. 2015; 35:340-350.

19. Garbers C, Aparicio-Siegmund S, Rose-John S. The IL-6/ gp130/STAT3 signaling axis: recent advances towards specific inhibition. Curr Opin Immunol. 2015; 34:75-82.

20. Ruffell B, Coussens LM. Macrophages and therapeutic resistance in cancer. Cancer Cell. 2015; 27:462-472.

21. Ostuni R, Kratochvill F, Murray PJ, Natoli G. Macrophages and cancer: from mechanisms to therapeutic implications. Trends in immunology. 2015; 36:229-239.

22. Noy R, Pollard JW. Tumor-associated macrophages: from mechanisms to therapy. Immunity. 2014; 41:49-61.

23. Little AG, Gay EG, Gaspar LE, Stewart AK. National survey of non-small cell lung cancer in the United States: epidemiology, pathology and patterns of care. Lung cancer. 2007; 57:253-260.

24. Antonov AV, Knight RA, Melino G, Barlev NA, Tsvetkov PO. MIRUMIR: an online tool to test microRNAs as biomarkers to predict survival in cancer using multiple clinical data sets. Cell Death Differ. 2013; 20:367.

25. Okayama H, Kohno T, Ishii Y, Shimada Y, Shiraishi K, Iwakawa R, Furuta K, Tsuta K, Shibata T, Yamamoto S, Watanabe S, Sakamoto H, Kumamoto K, et al. Identification of genes upregulated in ALK-positive and EGFR/KRAS/ ALK-negative lung adenocarcinomas. Cancer Res. 2012; 72:100-111.

26. Chitu V, Stanley ER. Colony-stimulating factor-1 in immunity and inflammation. Curr Opin Immunol. 2006; 18:39-48.

27. Saravanaperumal SA, Pediconi D, Renieri C, La Terza A. Skipping of exons by premature termination of transcription and alternative splicing within intron-5 of the sheep SCF gene: a novel splice variant. PLoS One. 2012; 7:e38657.

28. Espinosa I, Edris B, Lee $\mathrm{CH}$, Cheng HW, Gilks CB, Wang Y, Montgomery KD, Varma S, Li R, Marinelli RJ, West RB, Nielsen T, Beck AH, et al. CSF1 expression in nongynecological leiomyosarcoma is associated with increased tumor angiogenesis. Am J Pathol. 2011; 179:2100-2107.

29. Espinosa I, Beck AH, Lee CH, Zhu S, Montgomery KD, Marinelli RJ, Ganjoo KN, Nielsen TO, Gilks CB, West RB, van de Rijn M. Coordinate expression of colony-stimulating factor-1 and colony-stimulating factor-1-related proteins is associated with poor prognosis in gynecological and nongynecological leiomyosarcoma. Am J Pathol. 2009; 174:2347-2356.

30. Zhu Z, Shen Z, Xu C. Inflammatory pathways as promising targets to increase chemotherapy response in bladder cancer. Mediators Inflamm. 2012; 2012:528690.

31. Medrek C, Ponten F, Jirstrom K, Leandersson K. The presence of tumor associated macrophages in tumor stroma as a prognostic marker for breast cancer patients. BMC cancer. $2012 ; 12: 306$.

32. DeNardo DG, Brennan DJ, Rexhepaj E, Ruffell B, Shiao SL, Madden SF, Gallagher WM, Wadhwani N, Keil SD, Junaid SA, Rugo HS, Hwang ES, Jirstrom K, et al. Leukocyte complexity predicts breast cancer survival and functionally regulates response to chemotherapy. Cancer Discov. 2011; 1:54-67.

33. Allavena P, Mantovani A. Immunology in the clinic review series; focus on cancer: tumour-associated macrophages: undisputed stars of the inflammatory tumour microenvironment. Clin Exp Immunol. 2012; 167:195-205.

34. Joyce JA, Pollard JW. Microenvironmental regulation of metastasis. Nat Rev Cancer. 2009; 9:239-252.

35. Mantovani A, Allavena P, Sica A, Balkwill F. Cancer-related inflammation. Nature. 2008; 454:436-444.

36. Goswami S, Sahai E, Wyckoff JB, Cammer M, Cox D, Pixley FJ, Stanley ER, Segall JE, Condeelis JS. Macrophages promote the invasion of breast carcinoma cells via a colony-stimulating factor-1/epidermal growth factor paracrine loop. Cancer Res. 2005; 65:5278-5283.

37. Ai F, Zhang X, Li X, Qin Z, Ye Q, Tian L, Tang A, Li N, Li G, Ma J, Shen S. Up-regulation of matrix metalloproteinases in a mouse model of chemically induced colitis-associated cancer: the role of microRNAs. Oncotarget. 2015; 6:5412-5425. doi: 10.18632/oncotarget.3027.

38. Zhang X, Ai F, Li X, She X, Li N, Tang A, Qin Z, Ye Q, Tian L, Li G, Shen S, Ma J. Inflammation-induced S100A8 activates Id 3 and promotes colorectal tumorigenesis. Int $\mathbf{J}$ Cancer. 2015; 137:2803-2814.

39. Wen Q, Wang W, Chu S, Luo J, Chen L, Xie G, Xu L, Li M, Fan S. Flot-2 Expression Correlates with EGFR Levels and Poor Prognosis in Surgically Resected Non-Small Cell Lung Cancer. PLoS One. 2015; 10:e132190.

40. Luo Z, Dai Y, Zhang L, Jiang C, Li Z, Yang J, McCarthy JB, She X, Zhang W, Ma J, Xiong W, Wu M, Lu J, et al. miR-18a promotes malignant progression by impairing microRNA biogenesis in nasopharyngeal carcinoma. Carcinogenesis. 2013; 34:415-425.

41. Kouzmenko AP, Takeyama K, Ito S, Furutani T, Sawatsubashi S, Maki A, Suzuki E, Kawasaki Y, Akiyama T, Tabata T, Kato S. Wnt/beta-catenin and estrogen signaling converge in vivo. J Biol Chem. 2004; 279:40255-40258. 